\title{
Application of Risk Scoring System in Intra- operative Cell Salvage Patients: A Retrospective Study Based on Multicenter Data
}

BIN LYU ( $\sim$ hxlvbin@163.com )

West China Women's and Children's Hospital: Sichuan University West China Second University Hospital https://orcid.org/0000-0002-3922-4744

Xinghui Liu ( $\nabla$ hxxinghuiliu@163.com )

Daijuan Chen

Meng Chen

Jinhai Gou

\section{Research Article}

Keywords: intra-operative cell salvage, risk factor, scoring system, hemorrhage, cesarean section

Posted Date: March 29th, 2022

DOI: https://doi.org/10.21203/rs.3.rs-1241570/v2

License: (c) (1) This work is licensed under a Creative Commons Attribution 4.0 International License.

Read Full License 
1 Application of Risk Scoring System in Intra-operative Cell Salvage Patients: A

2 Retrospective Study Based on Multicenter Data

3 Bin Lyu ${ }^{1}$, Xinghui Liu ${ }^{1 *}$, Daijuan Chen ${ }^{1}$, Meng Chen ${ }^{1}$, Jinhai Gou ${ }^{1}$

$4 \quad{ }^{1}$ Key Laboratory of Birth Defects and Related Diseases of Women and Children (Sichuan

5 University), Ministry of Education, West China Second University Hospital of Sichuan

6 University, Chengdu, China.

7 *Corresponding author: Xinghui Liu, MD, Department of Obstetrics and Gynecology,

8 West China Second University Hospital of Sichuan University, Section 3 No. 20, Renmin

9 Road South, Chengdu 610041, China.

10 email: hxxinghuiliu@163.com. Phone number:(86)13980584568

Abstract

Background: Intraoperative cell salvage (IOCS) is widely used in cesarean section, and this study applied use of multi-center big data of IOCS in cesarean section to establish a bleeding risk assessment scale for IOCS patients. The purpose of this retrospective study was to explore the safety, health economy, and application

17 guidelines of IOCS in obstetrics.

Methods: We included 2621 patients who received IOCS technology during cesarean section in 12 hospitals between January 2012 - January 2020. The patients were divided into two groups, Group A, intraoperative blood loss $<1500 \mathrm{ml}$; Group B,

21 intraoperative blood loss $\geq 1500 \mathrm{ml}$. The clinical and imaging data associated with the 
risk of bleeding in patients taking IOCS were collected, and multivariable logistic regression analysis was conducted to develop a scoring system.

Results: All patients who received IOCS in our study had no serious adverse outcomes.

For the 2621 patients who take IOCS technology, while 407 cases not perform the blood transfusion, resulting in a waste rate of $15.5 \%$ for the resource. Multivariate logistic regression analysis was performed by selecting significant indicators from the univariate analysis and high-risk factors related to bleeding. The variables were maternal age $\geq 35$ years, number of cesarean sections, placental attachment position, and placenta previa, each scored 1 point; placenta accreta, blood pool in the placenta, abnormal retroplacental myometrium, placenta protruding to the anterior uterine wall and continuous disruption of the myometrium, each scored 2 points; and cervical canal invasion, scored 4 points. All were risk factors for intraoperative blood loss volume $\geq 1500 \mathrm{ml}$ in IOCS patients. A bleeding risk scoring system was constructed according to the above indicators. The area under the curve of the receiver operating characteristic curve (AUC) of the scoring system was 0.837 , and a total score of 5 points was identified as the optimal cut-off value, allowing good differentiation of intraoperative massive bleeding in IOCS patients (AUC, 0.837; 95\% confidence interval, $0.820-0.854$ ), along with a sensitivity of $81.2 \%$ and specificity of $75.3 \%$.

Conclusion: IOCS technology is a safe and effective measure to solve the problem of clinical blood source shortages in obstetric cesarean section. Moreover, the bleeding risk scoring system could better guide the clinical application of IOCS technology. 
Therefore, strictly mastering the clinical application guidelines of IOCS can save medical resources and reduce the economic burden on patients.

Keywords: intra-operative cell salvage; risk factor; scoring system; hemorrhage; cesarean section

\section{Background}

Intraoperative cell salvage (IOCS) is a technology that uses a blood-retrieving device to recover the patient's own hemorrhage during the operation, and the blood obtained after anticoagulation, washing, filtration, and concentration is returned to the patient [1]. In 1818, James Blundell et al. first used IOCS technology for pregnant women, however, the amniotic fluid may be present in the recovered blood, which could contaminate the blood and induce complications such as amniotic fluid embolism, fat embolism, and allogeneic immune response, thus limiting the application of IOCS in obstetrics [2-4]. However, with the implementation of the third-child policy, the number of high-risk pregnant women, with placenta previa, placenta accreta, and pregnancy-induced hypertension is increasing, and the incidence of obstetric hemorrhage is rising, leading to an increasing demand for allogeneic blood and straining the supplies of blood [5]. In addition, in our setting domestic obstetric blood transfusion mainly relies on non-remunerated blood donations, of which fresh or stock allogeneic blood is the main blood source, but adverse blood transfusion reactions of allergy, immunosuppression, and transmission of infectious diseases may be caused 
by allogeneic blood transfusion [6]. In recent years, the application of leukocyte filters can remove the amniotic fluid and squamous epithelial cell components from the recovered blood [7], and some studies have shown that the fetus's own red blood cells are very likely to enter the maternal-fetal circulation through the placenta during pregnancy, although the recovered autologous blood will also contain fetal red blood cells, and thus will not cause an immune response from the mother. [8]. A large number of clinical research guidelines including the National Institute for Health and Care Excellence, NICE [9], Association of Anesthetists, UK [10], and The American Society of Anesthesiologists [11]) all recommend that IOCS technology could be used in obstetric cesarean section.

Furthermore, it has been confirmed that the application of IOCS technology can promptly supplement the blood loss of pregnant women, maintain their blood volume and blood pressure, reduce the incidence of adverse blood transfusion reactions caused by allogeneic blood transfusion, and increase the success rate of rescuing pregnant women from massive bleeding during cesarean section [12,13]. IOCS has been widely used in cesarean sections in many domestic hospitals. This technology is predominantly used for patients at high risk of postpartum hemorrhage during cesarean section. As is known, postpartum hemorrhage is currently the leading cause of maternal death worldwide, accounting for $27.1 \%$ of maternal deaths [14].

Severe postpartum hemorrhage is a critical condition. For patients with severe postpartum hemorrhage, surgical hemostasis, uterine contraction drugs, and arterial intervention are crucial; meanwhile, blood transfusion is also a vital measure to rescue 
the lives of pregnant women $[15,16]$. However, the safety of allogeneic blood transfusions and the shortage of blood sources are becoming increasingly prominent. Although the screening system of blood donors and blood bank reserve technology have greatly improved the safety of blood transfusion, the occurrence of infectious diseases and other complications caused by allogeneic blood transfusion still cannot be completely avoided. Therefore, autologous blood transfusion has become an important way to solve the problem of clinical blood source shortages. Furthermore, it has been reported that the use of IOCS is widely used in cesarean section $[7,17,18]$, however, due to low recycling, it is not carried out to patients, resulting in a waste of resources. Therefore, the indications for the application of IOCS in cesarean section are still controversial, and the selection of the obstetric population requiring IOCS is an urgent problem that needs to be solved for clinicians. This retrospective cohort study collected clinical data for patients receiving IOCS during cesarean section in 12 tertiary hospitals across the country, and conducted a multivariable logistic regression analysis to develop a bleeding risk scoring system for evaluating the safety and health economy of IOCS and exploring the indications of the application of IOCS in obstetrics.

\section{Methods}

This retrospective study was approved by the Ethics Review Committee of West China Second University Hospital of Sichuan University (2017-033), and informed consent was obtained from all the included patients. All study methods of the 
retrospective study were conducted following the relevant regulations of a protocol approved by the Institutional Review Board of the West China Second University Hospital of Sichuan University. Of 2839 patients using IOCS technology who delivered in 12 tertiary hospitals between January 2012 and January 2020, 2621 cases were included in this study. All included patients had their pregnancies terminated by cesarean section, and IOCS technology was used during the cesarean section. Patients with serious medical and surgical diseases (liver diseases, kidney diseases, pregnancy complicated heart disease) and incomplete data were excluded from the study (Figure 1).

The Society for Maternal and Fetal Medicine recommends that the amount of postpartum hemorrhage $\geq 1500 \mathrm{ml}$ is the time for blood transfusion [19], and the 2621 cases were divided into two groups according to the intraoperative blood loss volume (1500 ml). A total of 1679 patients with blood loss volume $<1500 \mathrm{ml}$ was included in the control group (Group A), and 942 patients whose blood loss volume $\geq 1500 \mathrm{~mL}$ formed the massive bleeding group (Group B).

Clinical and imaging indicators of the 2621 patients were collected. Clinical indicators: Maternal characteristics were: maternal age, pre-parturient weight, height, gravidity, parity, number of cesarean sections, number of abortions, preoperative hemoglobin (HGB), history of uterine surgery, in vitro fertilization-embryo transfer (IVF-ET), twins, and antepartum hemorrhage. Newborn characteristics were: gestational age at delivery, newborn weight, Apgar score $(1 \mathrm{~min})$, neonatal death; ultrasonic indicators: placental attachment position, placenta previa, placenta accreta, 
blood pool in the placenta, boiling water sign in placenta, abnormal retroplacental myometrium, abnormal blood flow of retroplacental lacunae, cervical canal invasion, placenta protruding to the anterior uterine wall, continuous disruption of the posterior myometrium; Perioperative indicators: estimate blood loss (EBL), packed red blood cell (PRBC) transfusion, volume of autologous blood recovery/transfusion, fresh frozen plasma transfusion, platelet transfusion, cold precipitation transfusion, fibrinogen transfusion, cesarean hysterectomy rate, time of postoperative pyrexia, surgery time, delivery way, placenta management, pathological findings, postpartum adverse outcomes, total cost of allogeneic transfusion, and total cost of autologous transfusion.

\section{Statistical Analysis}

The collected data were analyzed by univariate analysis and multivariate analysis. Student's t-test, Mann-Whitney U-test, and chi-square test (X2 statistics) were used in the univariate analysis to assess the basic clinical features of the included patients. If the variables were normally distributed, an independent t-test was used for analysis, shown as the mean \pm standard deviation. The Mann-Whitney $U$ test was used for the analysis of non-normally distributed data, and the data are shown as the median (range). For categorical variables, relative frequencies were presented as the number/proportion (\%), which were calculated using the chi-square test. Furthermore, multivariable logistic regression was applied to analyze the association between the 
153

candidate risk factors and bleeding loss $(1500 \mathrm{ml})$ in patients who underwent IOCS technology.

Continuous variables were converted to binary variables, and the mean and median values of the indicators in the bleeding group (Group B) or the clinically recognized boundary values of the indicators were used as cut-off points. Each patient was scored by the odds ratio (OR) value corresponding to the risk factor in the scoring system, and a receiver operating characteristic curve (ROC) was drawn. The area under the curve (AUC) with 95\% confidence intervals and optimal cut-off value was calculated to evaluate the diagnostic efficacy of the scoring system. All statistical analyses were conducted using SPSS18.0 statistical software (IBM, Armonk, NY, USA). $\mathrm{P}$ values were shown for the assessment of indictors, and $\mathrm{P}<0.05$, Indicated a statistically significant difference.

\section{Results}

\section{Analysis of baseline characteristics between the two groups}

Of the 2621 pregnant women who underwent IOCS technology from 12 tertiary hospitals between January 2012 and January 2020, the baseline characteristics of Group A and Group B are summarized in Table 1. There were significant differences in maternal age, gravidity, parity, number of cesarean sections and abortions, history of uterine surgery, twins, antepartum hemorrhage, gestational age at delivery, and Apgar score $(1 \mathrm{~min})$ between the two groups $(\mathrm{P}<0.05)$. No significant differences 
were observed in terms of pre-parturient weight, height, preoperative HGB, IVF-ET, newborn weight, and neonatal death between groups A and $\mathrm{B}(\mathrm{P}>0.05)$ (Table 1).

\section{Analysis of ultrasound signs of two groups of patients}

The results of ultrasound signs between groups A and B are summarized in Table 2 . The proportions of placental attachment position, placenta previa, placenta accreta, blood pool in the placenta, boiling water sign in the placenta, abnormal retroplacental myometrium, abnormal blood flow of retroplacental lacunae, cervical canal invasion, placenta protruding to the anterior uterine wall, and continuous disruption of the posterior myometrium in group B were significantly higher than those in group A, (P $<0.05)$ (Table 2).

\section{Analysis of perioperative indexes in two groups}

Table 3 shows significantly higher estimated blood loss (EBL), usage of blood products (PRBCs, autologous blood recovery/transfusion, fresh frozen plasma, platelet, fibrinogen), cesarean hysterectomy rate, time of postoperative pyrexia, and surgery time in group $\mathrm{B}$ than in group A $(\mathrm{P}<0.05)$. There were statistically significant differences in placenta management, pathological findings, and postpartum adverse outcomes between the two groups $(\mathrm{P}<0.05)$. Most importantly, the total cost of allogeneic transfusion and total cost of autologous transfusion in group B were significantly higher than those in group $\mathrm{A},(\mathrm{P}<0.05)$, which indicated that strictly mastering the clinical application of IOCS is very important for patients (Table 3).

\section{Logistic multiple regression analysis for the risk factors of intraoperative} hemorrhage in IOCS patients 
We selected the statistical significance of univariate analysis indicators and associated risk factors for bleeding and included them in the multivariate analysis. Multivariable logistic regression analysis identified ten significant independent risk factors for intraoperative hemorrhage in IOCS patients. These were maternal age $\geq 35$ years, number of cesarean sections, placental attachment position, placenta previa, placenta accreta, blood pool in the placenta, abnormal retroplacental myometrium, placenta protruding to the anterior uterine wall, cervical canal invasion, and continuous disruption of the myometrium. Therefore, we selected these ten potential risk factors for the scoring system, which was used to assess the bleeding risk. The detailed results of the multivariate analysis are presented in Table 4.

According to the OR values of eight risk factors developed from the multiple logistic regression analysis, a risk scoring system for bleeding for IOCS patients was established, and the OR values were converted into corresponding points. These are detailed as: maternal age $\geq 35$ years old ( 1 point), number of cesarean sections ( 1 point), placental attachment position (1 point), placenta previa (1 point), placenta accreta ( 2 points), blood pool in the placenta ( 2 points), abnormal retroplacental myometrium (2 points), Placenta protruding from the anterior uterine wall (2 points), and continuous disruption of the myometrium ( 2 points) and cervical canal invasion ( 4 points), were the risk factors of intro-operative blood loss volume $\geq 1500 \mathrm{ml}$ in IOCS patients (Table 5).

Then, a bleeding risk scoring system was constructed according to the above indicators, and the total scores ranged from to $0-18$ points, with an optimal threshold 
of 5 points. The area under curve (AUC) of the receiver operating characteristic curve of the scoring system was 0.837 , and a total score of 5 points was identified as the optimal cut-off value, allowing good differentiation of intraoperative massive bleeding in IOCS patients (AUC, 0.837; 95\% confidence interval [CI], 0.820-0.854). The relatively high sensitivity $(81.2 \%)$ and specificity $(75.3 \%)$, showed good efficacy in differentiating the risk of bleed loss $\geq 1500 \mathrm{ml}$ in IOCS patients (Figure 2).

\section{Discussion}

Obstetric hemorrhage is currently the leading cause of maternal death, especially in patients with massive hemorrhages [20,21]. Timely and reasonable blood transfusion is one of the most effective measures to reduce maternal mortality, and has been widely performed in obstetric operations [22]. The clinical use of fresh or stocked allogeneic blood has become the main source of blood transfusion therapy, while the safety of allogeneic blood transfusion and the increasing tension of blood sources have attracted attention from medical workers. Although improvements in the blood donor screening system and the storage technology of blood banks have greatly improved the safety of blood transfusion, the occurrence of blood transfusion-related allergic reactions cannot be completely avoided $[23,24]$. Therefore, reasonable, safe, and effective blood availability has become an urgent problem.

Intraoperative cell salvage (IOCS) is a blood source protective measure that has gradually become popular in recent years. This is the most effective method for blood transfusion to reduce blood-borne diseases related to blood transfusions [25]. IOCS is 
a technology that takes the patient's intraoperative bleeding and blood accumulated in the body cavity and this blood is filtered by multiple layers into the blood recovery tank; after separation, washing, purification, the free hemoglobin, cell debris, fat droplets, and other impurities are diverted into the large waste liquid bag to reduce the residual pollutants, and finally the concentrated red blood cell suspension is returned to the patient [26]. With the use of a leukocyte removing filter, the amniotic fluid components from the recovered blood can be almost completely eliminated [18,27].

Although multiple studies with a large number of clinical samples have confirmed the safety of this technology, there are still some taboos in promoting this technique $[2,28]$. In particular, the safety and effectiveness of IOCS during cesarean section is still controversial [4], and previous studies have reported inconsistent conclusions about the application of IOCS in cesarean section on allogeneic transfusion and the economic benefits to obstetric patients $[13,29]$. Currently, IOCS technology has been widely used in obstetrics, and some scholars have reported that IOCS technology is an effective method of reducing transfusion for cases at high risk of obstetric hemorrhage, and which is economically reasonable, while for routine cesarean deliveries, IOCS is not recommended $[12,30]$. Therefore, it is necessary to discuss the application guidelines to provide evidence as to the rationale for such use and to increase confidence in its use as an effective application in obstetrics.

In our study, 2621 patients were included, and all patients who received IOCS had no serious adverse outcomes of e.g., amniotic fluid embolism and maternal death; therefore, IOCS is considered relatively safe and economical. However, this study 
showed that $407(15.5 \%)$ cases not perform the blood transfusion, because some patients had a small amount of intraoperative recovered blood. This could not reach the reinfusion standard and did not undergo reinfusion, which caused a waste of resources to a certain extent. Therefore, we conducted a multi-factor analysis to explore the clinical application guidelines of IOCS in obstetrics. Multivariate logistic regression analysis was performed by selecting statistically significant indicators in univariate analysis and high-risk factors related to bleeding to construct a bleeding risk scoring system for guiding the rational use of IOCS technology. This identified ten significant independent risk factors, namely, maternal age $\geq 35$ years, number of cesarean sections, placental attachment position, placenta previa, placenta accreta, blood pool in the placenta, abnormal retroplacental myometrium, placenta protruding to the anterior uterine wall, cervical canal invasion, and continuous disruption of the myometrium. Therefore, the above 10 variables were included in the scoring system to predict the risk of intraoperative hemorrhage in patients with IOCS. The results showed that the area under the curve (AUC) of the receiver operating characteristic curve for the scoring system was 0.837 , and a total score of 5 points was identified as the optimal cut-off value allowing good differentiation of intraoperative massive bleeding in IOCS patients. Consequently, IOCS technology was not shown to be beneficial for all obstetric patients. From our study, a total score $\geq 5$ points indicating a high risk of intraoperative hemorrhage, was recommended to use the IOCS technology. 

leukocyte filters, IOCS technology has been widely used in obstetric cesarean section,

285 while there is no recommended application guide in the literature about the 286 technology, which leads to excessive use of IOCS and a waste of resources. In our research, we conducted a multivariate analysis in obstetrics patients using the IOCS, designed a bleeding assessment scale, and achieved better and more reasonable usage of IOCS technology, which can to a certain extent, reduce the economic burden on patients. Second, this study focused on patients who used IOCS technology in cesarean section. Finally, the scoring system not only included clinical data, but also included ultrasonographic indicators, which play an important role in the evaluation

293 of hemorrhagic disease (placenta previa, placenta accreta, and vasa previa) in clinical practice. Therefore, a good understanding of the clinical application 
save medical resources and reduce the economic burden on patients. operating characteristic curve

\section{Abbreviations}

AUC: Area under the curve; CI: Confidence interval; EBL: Estimated blood loss; HGB: Hemoglobin; IOCS: Intraoperative cell salvage; IVF- ET: In-vitro fertilization embryo transfer: OR: Odds ratio; PRBC: Packed red blood cells; ROC: Receiver

\section{Acknowledgements}

We thank the pregnant women who participated in our study as well as the research coordinators and assistants from the project for their contribution.

Thanks to the following hospitals for providing clinical study data: West China Second University Hospital of Sichuan University 、 The First Affiliated Hospital of Chongqing Medical University 、 Obstetrics and Gynecology Hospital of Fudan University 、 The Second Hospital of HeBei Medical University 、 Tongji Hospital affiliated to Tongji Medical College of Huazhong University of Science and Technology 、 Union Hospital affiliated to Tongji Medical College of Huazhong University of Science and Technology 、The Fourth Hospital of Shijiazhuang、Xiamen maternal and Child Health Hospital、Women's Hospital School Of Medicine Zhejiang University 、 The First Affiliated Hospital of Zhengzhou University 、 The Third Affiliated Hospital of Zhengzhou University 、 Ningbo women's and children's 
Hospital.

\section{Authors' contribution}

$\mathrm{BL}$ and $\mathrm{XL}$ contributed to the conception and design of the study. MC, BL, JG and

DC contributed to data collection, data analysis, and manuscript writing. BL and XL provided the financial support; BL and XL revised the manuscript. All authors read and approved the final version of the manuscript. corresponding author on reasonable request. University Hospital of Sichuan University (2017-033), and informed consent was

\section{Availability of data and materials}

The datasets used and/or analyzed during the current study are available from the

\section{Declarations}

\section{Ethical approval and consent to participate}

This was obtained from the Ethics Review Committee of West China Second obtained from all the included patients. All study methods of the retrospective study 
were conducted in accordance with relevant regulations of a protocol approved by the

350 Institutional Review Board from the West China Second University Hospital of

351 Sichuan University. All tests used in this study could be administered free of charge

352 for non-commercial research and educational purpose. Permission was granted by

353 original authors when needed.

\section{Consent for publication}

356 Not applicable.

\section{Competing interests}

360 The authors declare no competing interests.

\section{Author details}

1 Department of Obstetrics and Gynecology, West China Second University Hospital of Sichuan University, Chengdu, China .2 Key Laboratory of Birth Defects and Related Diseases of Women and Children (Sichuan University), Ministry of Education, West China Second University Hospital of Sichuan University, Chengdu, 367 China 


\section{References}

372 1.Goucher, H. , Wong, C. A. , Patel, S. K. , \& Toledo, P. . (2015). Cell salvage in obstetric

373 s. Anesthesia \& Analgesia, 121(2), 465.https://doi.org/10.1213/ane.000000000000078

374 2. Liu, Y., Li, X., Che, X., Zhao, G., \& Xu, M. (2020). Intraoperative cell salvage for obstetrics: a

375 prospective randomized controlled clinical trial. BMC Pregnancy and Childbirth, 20(1), 1-8.

376 https://doi.org/10.1186/s12884-020-03138-w

377 3. Nash, Z. J., Kunde, K., \& Mascarenhas, L. J. (2014). The role of intraoperative cell salvage in

378 abdominal myomectomy. American Journal of Obstetrics \& Gynecology, 211(4), 440-441.

379 https://doi.org/10.1016/j.ajog.2014.05.019

380 4.Grainger, H., \& Catling, S. (2018). Intraoperative cell salvage in obstetrics. Journal of

381 Perioperative Practice, 28(3), 51-58. https://doi.org/10.1177/1750458918755960

382 5.Konig, G. (2018). Recommendations on blood recovery in obstetrics. In Transfusion Management

383 of the Obstetrical Patient (pp. 67-72). Springer,Cham.

384 https://doi.org/10.1007/978-3-319-77140-3_7

385 6.Simancas - Racines, D., Osorio, D., Martí - Carvajal, A. J., \& Arevalo - Rodriguez, I. (2015).

386 Leukoreduction for the prevention of adverse reactions from allogeneic blood

387 transfusion. Cochrane Database of Systematic Reviews, (12).

388 https://doi.org/10.1002/14651858.cd009745.pub2

389 7.McDonnell, N. J., Kennedy, D., Long, L. J., Gallagher-Swann, M. C., \& Paech, M. J. (2010). The

390 development and implementation of an obstetric cell salvage service. Anaesthesia and intensive

391 care, 38(3), 492-499.https://doi.org/10.1177/0310057×1003800313

392 8.Ralph, C. J., Sullivan, I., \& Faulds, J. (2011). Intraoperative cell salvaged blood as part of a blood

393 conservation strategy in Caesarean section: is fetal red cell contamination important?. British

394 journal of anaesthesia, 107(3), 404-408.https://doi.org/10.1093/bja/aer168

395 9.No, R. GUIDANCE FOR THE PROVISION OF INTRAOPERATIVE CELL SALVAGE GUIDANCE

396 FOR THE PROVISION OF INTRAOPERATIVE CELL SALVAGE. Blood. gov. au.

397 10.Klein, A. A., Bailey, C. R., Charlton, A. J., Evans, E., Guckian - Fisher, M., McCrossan, R., ... \&

398 Torella, F. (2018). Association of Anaesthetists guidelines: cell salvage for peri - operative blood

399 conservation 2018. Anaesthesia, 73(9), 1141-1150.https://doi.org/10.1111/anae.14331 
401 11.Practice Guidelines for Obstetric Anesthesia. (2016). An updated report by the American Society

402 of Anesthesiologists task force on obstetric anesthesia and the society for obstetric anesthesia and

403 perinatology. Anesthesiology, 124(2),270-300.https://doi.org/10.1097/01.aoa.0000504699.59827.5

$404 \quad 6$

405 12.Lim, G., Melnyk, V., Facco, F. L., Waters, J. H., \& Smith, K. J. (2018). Cost-effectiveness

406 analysis of intraoperative cell salvage for obstetric hemorrhage. Anesthesiology, 128(2),

407 328-337.https://doi.org/10.1097/aln.0000000000001981

408 13.Zeng, K., Huang, W., Yu, C., \& Wang, R. (2018). How about "The effect of intraoperative cell

409 salvage on allogeneic blood transfusion for patients with placenta accreta"?: an observational

410 study. Medicine, 97(22).https://doi.org/10.1097/md.0000000000010942

411 14.Say, L., Chou, D., Gemmill, A., Tunçalp, Ö., Moller, A. B., Daniels, J., ... \& Alkema, L. (2014).

412 Global causes of maternal death: a WHO systematic analysis. The Lancet global health, 2(6),

413 e323-e333.https://doi.org/10.1016/s2214-109x(14)70227-x

414 15.O'Brien, K. L., Shainker, S. A., \& Lockhart, E. L. (2018). Transfusion management of obstetric

415 hemorrhage. Transfusion Medicine Reviews, 32(4),249-255.

416 https://doi.org/10.1016/j.tmrv.2018.05.003

417 16.Patterson, J. A., Roberts, C. L., Bowen, J. R., Irving, D. O., Isbister, J. P., Morris, J. M., \& Ford, J.

418 B. (2014). Blood transfusion during pregnancy, birth, and the postnatal period. Obstetrics \&

419 Gynecology, 123(1), 126-133.https://doi.org/10.1097/aog.00000000000000054

420 17.Elagamy, A., Abdelaziz, A., \& Ellaithy, M. (2013). The use of cell salvage in women undergoing

421 cesarean hysterectomy for abnormal placentation. International Journal of Obstetric

422 Anesthesia, 22(4), 289-293.https://doi.org/10.1016/j.ijoa.2013.05.007

423 18.Rogers, W. K., Wernimont, S. A., Kumar, G. C., Bennett, E., \& Chestnut, D. H. (2013). Acute

424 hypotension associated with intraoperative cell salvage using a leukocyte depletion filter during

425 management of obstetric hemorrhage due to amniotic fluid embolism. Anesthesia \&

426 Analgesia, 117(2), 449-452.https://doi.org/10.1213/ane.0b013e3182938079

427 19.Shields, L. E., Smalarz, K., Reffigee, L., Mugg, S., Burdumy, T. J., \& Propst, M. (2011).

428 Comprehensive maternal hemorrhage protocols improve patient safety and reduce utilization of

429 blood products. American journal of obstetrics and gynecology, 205(4), 368-e1. 
https://doi.org/10.1016/j.ajog.2011.06.084

20.Baird, E. J. (2017). Identification and management of obstetric hemorrhage. Anesthesiology clinics, 35(1), 15-34.https://doi.org/10.1016/j.anclin.2016.09.004

21.Reale, S. C., Easter, S. R., Xu, X., Bateman, B. T., \& Farber, M. K. (2020). Trends in postpartum hemorrhage in the United States from 2010 to 2014. Anesthesia \& Analgesia, 130(5), e119-e122. https://doi.org/10.1213/ane.0000000000004424 22.Dhariwal, S. K., Khan, K. S., Allard, S., Wilson, M., \& Moore, P. (2014). Does current evidence support the use of intraoperative cell salvage in reducing the need for blood transfusion in caesarean section?. Current opinion in obstetrics \& gynecology, 26(6), 425. https://doi.org/10.1097/gco.0000000000000116

23. Liumbruno, G. M., Liumbruno, C., \& Rafanelli, D. (2011). Intraoperative cell salvage in obstetrics: is it a real therapeutic option?. Transfusion, 51(10), 2244-2256. https://doi.org/10.1111/j.1537-2995.2011.03116.x 24. Milne, M. E., Yazer, M. H., \& Waters, J. H. (2015). Red blood cell salvage during obstetric hemorrhage. Obstetrics \& Gynecology, 125(4), 919-923. https://doi.org/10.1097/aog.0000000000000729 25.DeAndrade, D., Waters, J. H., Triulzi, D. J., Alarcon, L., Wisniewski, M. K., Dyga, R., \& Yazer, M. H. (2016). Very low rate of patient - related adverse events associated with the use of intraoperative cell salvage. Transfusion, 56(11), 2768-2772.https://doi.org/10.1111/trf.13791 cesarean delivery. International journal of obstetric anesthesia, 41, 59-64. https://doi.org/10.1016/j.ijoa.2019.07.003 27. Mei, K., Du, L., Yan, M., Zhang, Z., Zhang, F., Gong, L., ... \& Liu, J. (2015). Modified leukocyte filter removes tumor cells from the salvaged blood. PloS one, 10(6), e0130864. https://doi.org/10.1371/journal.pone.0130864 28. Khan, K. S., Moore, P., Wilson, M., Hooper, R., Allard, S., Wrench, I., ... \& Dodds, J. (2018). A randomised controlled trial and economic evaluation of intraoperative cell salvage during caesarean section in women at risk of haemorrhage: the SALVO (cell SALVage in Obstetrics) trial. Health Technology Assessment (Winchester, England), 22(2), 1. 
460 https://doi.org/10.3310/hta22020

461 29. Yan, H., Hu, L. Q., Wu, Y., Fan, Q., Wong, C. A., \& McCarthy, R. J. (2018). The association of 462 targeted cell salvage blood transfusion during cesarean delivery with allogeneic packed red blood

463 cell transfusions in a maternity hospital in China. Anesthesia \& Analgesia, 127(3), 706-713.

464 https://doi.org/10.1097/01.aoa.0000552917.58695.28

465 30.Wu, X., Yao, S. L., Wu, J., Li, C. Y., \& Xia, L. M. (2020). Intra-operative cell salvage for cesarean

466 delivery: a retrospective study using propensity score matched analysis. Chinese medical

467 journal, 133(02), 183-189.https://doi.org/10.1097/cm9.0000000000000620

468

469

470

471

472

473

474

475

476

477

478

479 


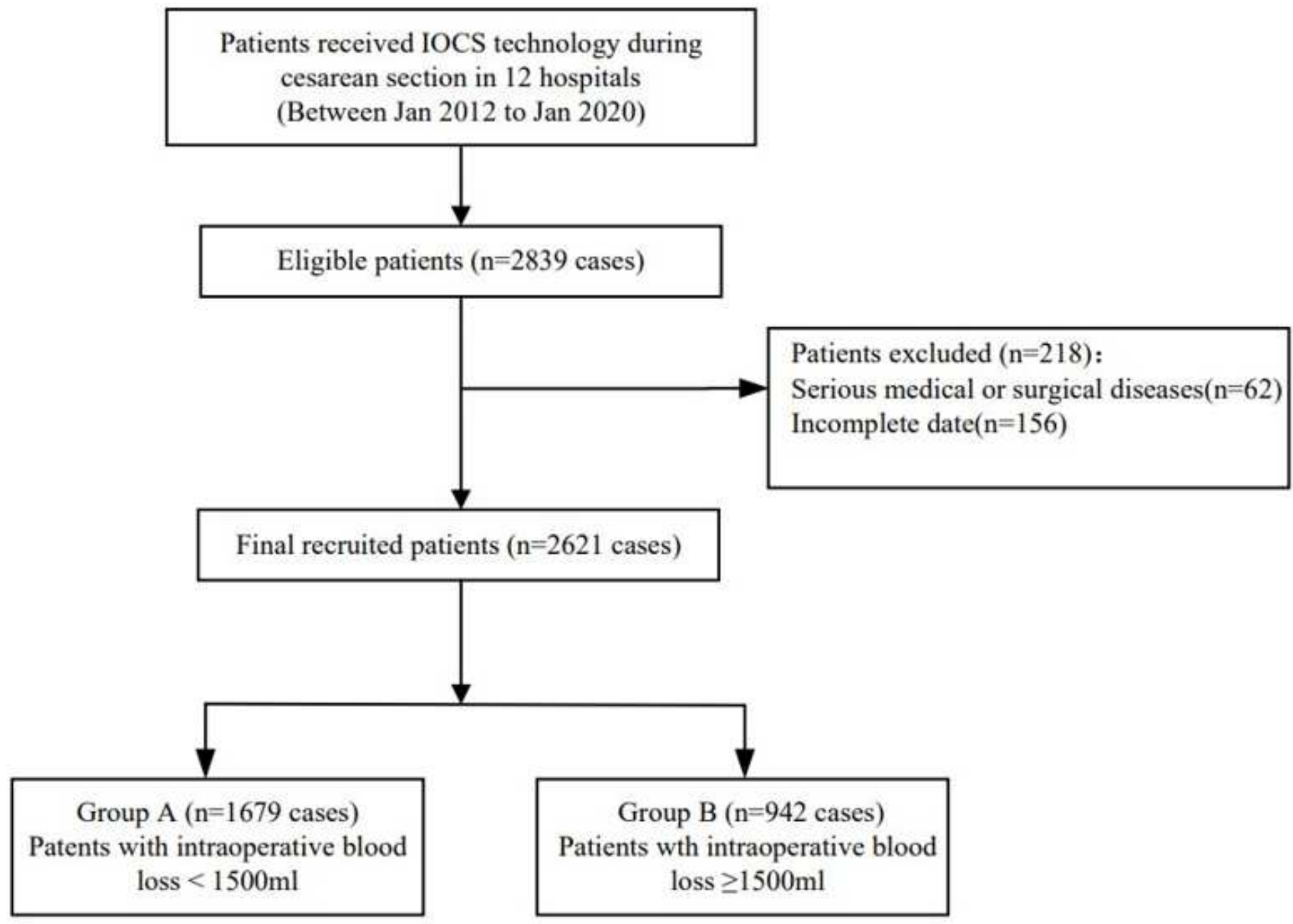

\section{Figure 1}

Flowchart of selection of the patients for the studyFlowchart of selection of the patients for the study 


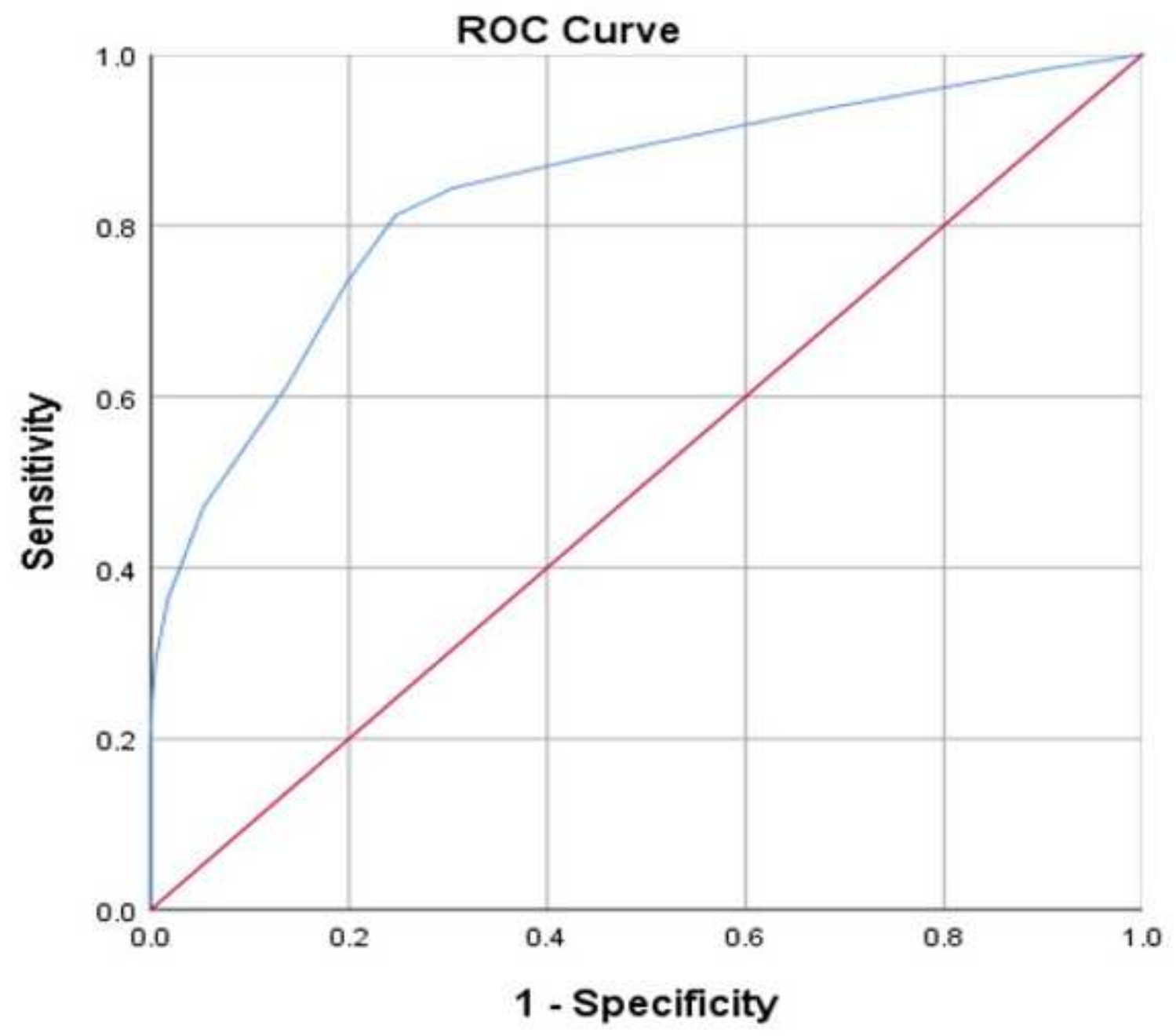

Figure 2

The ROC curve of risk scoring system of bleeding for IOCS patients. 